\title{
Antimicrobial stewardship of Chinese ministry of health reduces multidrug- resistant organism isolates in critically ill patients: a pre-post study from a single center
}

Xudong Ma ${ }^{1 \dagger}$, Jianfeng Xie ${ }^{2 \dagger}$, Yi Yang${ }^{2}$, Fengmei Guo ${ }^{2}$, Zhiwei Gao², Hua Shao ${ }^{3}$, Yingzi Huang ${ }^{2}$, Congshan Yang ${ }^{2}$ and Haibo Qiü ${ }^{2 *}$

\begin{abstract}
Background: China's Ministry of Health $(\mathrm{MOH})$ has established a policy about the antimicrobial stewardship. To date, the effects of this policy on multidrug-resistant organism (MDRO) in critically ill patients are unknown.

Methods: A pre-post study was conducted on intensive care unit (ICU) patients from June 2010 to May 2011 and from June 2012 to May 2013. Bacterial cultures were conducted at ICU admission and discharge. In June 2011, our hospital started to administer the antimicrobial stewardship program of Chinese $\mathrm{MOH}$. We collected the data on antimicrobial consumption during the 3-year period in all hospital and individual department every month, and analyzed the correlation between the proportion of critically patients colonized or infected with MDRO and antimicrobial consumption.
\end{abstract}

Results: A total of 978 patients were involved in the present study. With the intervention, the monthly mean Defined Daily Dose (DDD) per 100 occupied bed-days throughout the hospital decreased from $96 \pm 7$ to $65 \pm 6$ $(p<0.001)$, and the proportion of patients colonized or infected with MDRO decreased from 36 to $13 \%$ at the time of ICU admission and declined from 48 to $29 \%$ at the time of ICU discharge (both $p<0.001$ ). There was a significant positive relationship between the proportion of all critically ill patients colonized or infected with MDRO at ICU admission and the DDD of the entire hospital $\left(R^{2}=0.7858, p<0.001\right)$.

Conclusion: The antimicrobial stewardship program of Chinese $\mathrm{MOH}$ reduced the consumption of antibiotics. Moreover, the proportion of patients colonized or infected with MDRO decreased along with reduced consumption of antibiotics.

Trial registration: Retrospectively registered: NCT02128399; Date of registration: 22 APR 2014; Detail information web link: https://clinicaltrials.gov/ct2/show/NCT02128399?term=NCT02128399\&rank=1

Keywords: Antimicrobial stewardship, Antimicrobial consumption, Defined daily dose, Multidrug-resistant organism

\footnotetext{
* Correspondence: haiboq2000@163.com

${ }^{\dagger}$ Equal contributors

2Department of Critical Care Medicine, Nanjing Zhongda Hospital, School of

Medicine, Southeast University, Nanjing 210009, China

Full list of author information is available at the end of the article
} 


\section{Background}

Antimicrobial exposure increases the selection for various drug-resistant organisms [1]. Numerous studies have demonstrated the association between antimicrobial use and MDRO detection [2-4]. However, to our knowledge, there are few data to support the concept that reducing antibiotic use actually leads to improvements in antibiotic susceptibilities [1]. Moreover, antimicrobial stewardship was demonstrated to reduce MDRO and was strongly recommended in clinics [5]. The World Health Organization (WHO) strongly recommends that governments implement antimicrobial stewardship programs directed at containing antimicrobial resistance [6]. However, a considerable gap exists between theory and clinical practice [7]. Antibiotic use is increasing even in some countries such as Norwegian with a low antibiotic resistance rate $[8,9]$.

In China, the overuse of antibiotics is common [10], and the prevalence of antimicrobial resistance isolates is higher than that in North America and Europe [11]. Data reported by the Chinese Ministry of Health $(\mathrm{MOH})$ National Antimicrobial Resistance Investigation Net (Mohnarin) indicates that antimicrobial resistance is rising steadily [12]. In 2011, Chinese MOH established policies concerning the antimicrobial stewardship to improve the intelligent use of antibiotics. Most hospitals in China gradually implemented the policies. These policies included restricting the kinds of antibiotics, setting the targets for antibiotic prescription in hospitalized patient and prophylactic use of antibiotics in clean operations (Please see the detail of polices in methods of Antimicrobial stewardship). However, to the best of our knowledge, no studies have shown whether the antimicrobial stewardship can reduce infection or colonization with MDRO in critically ill patients. Therefore, in the present study, we aimed to elucidate the role of the antimicrobial stewardship on the antimicrobial consumption and infection or colonization with MDRO in critically ill patients.

\section{Methods}

\section{Study design}

The pre-post study was performed in the ICU of a university-affiliated hospital. The protocol was approved by Nanjing Zhongda Hospital Institutional Ethics Committee (Approval Number: 2011ZDLL012.0). Written informed consent was obtained from the patients or next of kin. The trial was registered at clinicaltrials.gov (NCT02128399).

The study was divided into two periods according to the intervention of antibiotics management policy, which started in June 2011. Phase 1 was defined as one year before the intervention (from June 2010 to May 2011). Phase 2 was defined as one year after the intervention (from June 2012 to May 2013). During the two phases of the study, all patients hospitalized in the ICU and patients discharged from ICU before the end of the two studied phases were eligible to participate in the study. We only selected the data of patients for their first admission to ICU. Screening for MDRO was performed in our ICU as part of an infection control policy, and microbiological cultures of different specimens were performed according to clinical status. Bacterial cultures from nasal swabs, lower respiratory tract secretions and the infected area were performed at the time of ICU admission and discharge.

We performed the same policy of screen during the two study periods. We also had taken executive infection control measures during this pre-post study period in our hospital. All of the patients were treated by the attending physician.

\section{Antimicrobial stewardship}

According to the protocol of antibiotic administrative group, our hospital was directed to implement the protocol in June 2011. The policy included antibiotic procurement was restricted to 50 agents in our hospital from June 2011. Meanwhile, targets for antibiotic prescription are set at less than 60 and $20 \%$ of all prescriptions for hospitalized patients and outpatients, respectively. Prophylactic use of antibiotics in clean operations should be lowered to $30 \%$ of patients and reduced to less than 24 h' duration; and antibiotic utilization in hospitalized patients should be less than 40 Defined Daily Dose (DDD) per 100 patient; rationality of antibiotic use, which includes timing, duration and appropriate medications should be more than $90 \%$. In our hospital, special antimicrobial agents, such as carbapenem, glycopeptide, linezolid, daptomycin, and tigecycline, must be approved by a designated doctor of pharmacy; in particular case, such as during salvage, doctors are permitted to urgently use these special antimicrobial no more than one day. The trained pharmacists and infection preventionists were all part of the antimicrobial stewardship in our hospital. They were responsible for monitoring these medications, and every month, they announced the antimicrobial agents in every clinic and department. If the individual department did not reach the targets which mentioned, the director of the department would provide the reason and be warned by the hospital. If there was no desirable reason why they could not achieve the targets, the salary of all the staff of the department would be deducted. During the study period, we practiced infection control measures according to the recommendations of the Centers for Disease Control (CDC) of the USA [13].

\section{Data collection and definitions}

Beginning in June 2011, the management of antibacterial drugs in clinical applications was implemented in every 
department in our hospital. For each month, we recorded the aggregate data on active surveillance testing and antibiotic consumption. We prospectively collected all data concerning patient characteristics at the time of ICU admission and during the patients' ICU stay. We collected information about ICU patients' microbiological culture results and antibiotic consumption between June 2010 and the end of May 2011. The antibiotic consumption data were obtained from the hospital computer center database.

We used the Defined Daily Dose DDD per 100 occupied bed-days to indicate antibiotic consumption in our hospital and every department. The DDD for adults was obtained from the anatomical therapeutic chemical (ATC) classification index from the WHO, with the DDD unit expressed in grams.

MDRO were defined as bacteria were resistant to at least three antimicrobial classes which included methicillin-resistant Staphylococcus aureus (MRSA), vancomycin-resistant enterococci (VRE), Pseudomonas aeruginosa, Acinetobacter baumannii, and extendedspectrum $\beta$-lactamase (ESBL)-producing or carbapenemaseproducing gram-negative bacilli [14]. We use laboratory tests to determine these MDRO. Minimal Inhibitory Concentration (MIC) method was used to determine the bacterial drug resistance. MRSA was defined as Staphylococcus aureus resistant to oxacillin and VRE was defined as enterococci resistant to vancomycin. ESBL-producing organisms were defined as Gram-negative bacilli resistant to ceftazidime but sensitive to enzyme inhibitor such as piperacillintazobactam.

\section{Statistical analysis}

All of the statistical analyses were conducted using SPSS 13.0 software (release 13.0, SPSS, Chicago, IL, USA). The distribution of quantitative variables was tested. Normally and abnormally distributed quantitative variables are presented as the mean \pm standard deviation and the median (25th-75th interquartile range), respectively. All $p$ values were two-tailed. A $p<0.05$ was considered to be statistically significant.

We assessed the differences in categorical variables using the $\chi^{2}$ test or Fisher's exact test. We analyzed the differences in length of ICU stay and stay until hospital discharge or death using the Mann-Whitney $U$ test.

Antibiotic consumption and infection or colonization with MDRO were assumed to be independent variables; the association between the 2 variables was evaluated using the parametric Pearson's or non-parametric Spearman's correlation coefficient.

\section{Results}

\section{General condition}

A total of 978 patients were involved in our study, among whom 433 patients were involved before the intervention and 545 patients were involved after the antibacterial drug clinical application management. The patient characteristics are presented in Table 1 . Of the 978 patients, the median length of the ICU stay was 5 (2-10) days, and the median length of the hospital stay was 18 (8-30) days. One hundred fifty-six (24\%) patients died in the ICU, and the hospital mortality rate was $26 \%$ (Table 1). There were no significant differences in ICU mortality, hospital mortality and length of ICU stay between both phases; however, the length of the hospital stay was reduced after the intervention (17 days vs. 18 days, $p=0.009$ ).

\section{Antimicrobial consumption}

The monthly antimicrobial consumption throughout the hospital from June 2010 to May 2013 is shown in Fig. 1. With the antimicrobial stewardship program in clinical applications, we found that the rational use of antibiotics had improved. In the preventative use of antibiotics in

Table 1 Summary of patient characteristics

\begin{tabular}{lllll}
\hline & All patients (978) & Before management (433) & After Management (545) & $p$ value \\
\hline Age, years & $64 \pm 19$ & $64 \pm 19$ & $65 \pm 18$ & $363(67)$ \\
Male (\%) & $630(64)$ & $267(62)$ & $16 \pm 9$ & 0.319 \\
APACHEll score & $16 \pm 9$ & $16 \pm 9$ & & 0.109 \\
Admission source & & & $181(33)$ & $268(49)$ \\
$\quad$ Emergency & $313(32)$ & $132(30)$ & $96(18)$ & 0.578 \\
$\quad$ Surgery & $473(48)$ & $205(47)$ & $132(24)$ & 0.268 \\
$\quad 192(20)$ & $96(22)$ & $148(27)$ & 0.569 \\
ICU modicine & $244(25)$ & $112(26)$ & $5(2-10)$ & 0.075 \\
Hospital mortality (\%) & $281(29)$ & $133(31)$ & $17(9-30)$ & 0.55 \\
Duration of ICU stay & $5(2-10)$ & $18(8-33)$ & 0.954 \\
Duration of hospitalization & $17(8-30)$ & &
\end{tabular}




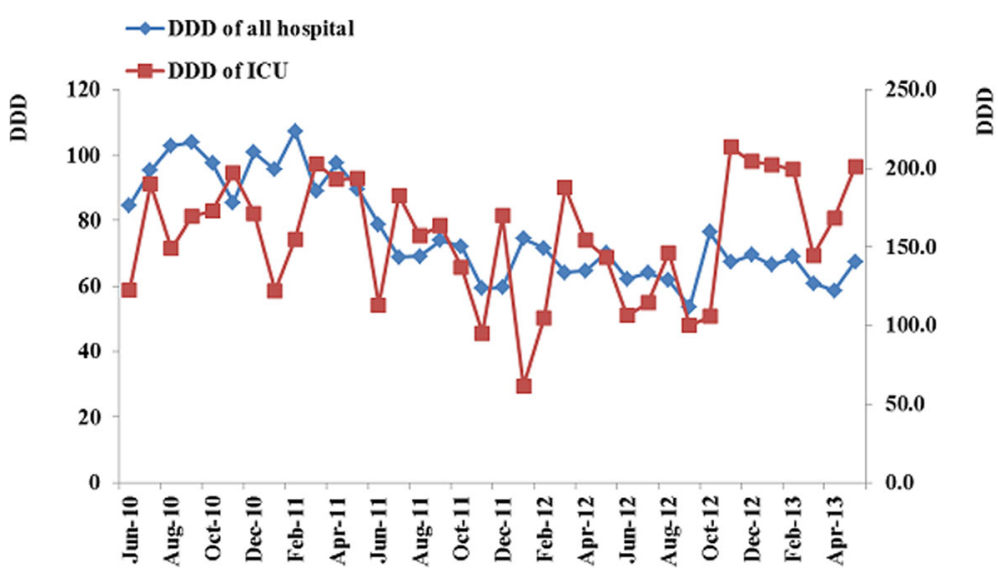

Fig. 1 Antibiotic consumption of our hospital and ICU decreased after the management of antibacterial drugs in clinical applications

clean operation, for example, the percentage decreased from nearly 100 to $35 \%$. The DDD per 100 occupied beddays in our hospital decreased from 96 before intervention to 65 after intervention (Table 2). In most departments, the DDD per 100 occupied bed-days decreased similarly, and only in the thoracic surgery, neurology and urology departments, the DDD per 100 occupied bed-days did not decrease from 2010 to 2013 (Table 2).

The monthly antimicrobial consumption in the ICU is shown in Fig. 1. The DDD per 100 occupied bed-days in the ICU during the study period was higher than that observed in other departments. With the antimicrobial stewardship, the DDD per 100 occupied bed-days also decreased from $170 \pm 28$ to $159 \pm 44$ in ICU $(p=0.018)$.

During the antimicrobial stewardship, the category of antibiotics did not change (Additional file 1: Table S1). The median duration of antibiotics were $3.64(1.96,6.18)$ and $3.42(1.65,6.19)$ before and after antimicrobial stewardship, respectively. In addition, the dose of antibiotics also did not change during the intervention.

Table 2 Mean monthly DDD per 100 occupied bed-days before and after the antimicrobial stewardship

\begin{tabular}{lcll}
\hline Department & $\begin{array}{l}\text { Before } \\
\text { management }\end{array}$ & $\begin{array}{l}\text { After } \\
\text { management }\end{array}$ & $p$ value \\
\hline All & $96 \pm 7$ & $65 \pm 6$ & $<0.001$ \\
Neurosurgery & $78 \pm 26$ & $45 \pm 17$ & 0.002 \\
Thoracic surgery & $96 \pm 20$ & $106 \pm 36$ & 0.38 \\
General surgery & $115 \pm 16$ & $93+20$ & 0.003 \\
Urology surgery & $117 \pm 15$ & $129 \pm 10$ & 0.02 \\
Orthopedics & $82 \pm 9$ & $40+7$ & $<0.001$ \\
Respiratory medicine & $178 \pm 18$ & $128+15$ & $<0.001$ \\
Neurology & $36 \pm 9$ & $35 \pm 12$ & 0.963 \\
Cardiology & $74 \pm 12$ & $35 \pm 6$ & $<0.001$ \\
ICU & $170 \pm 28$ & $159 \pm 44$ & 0.018 \\
\hline
\end{tabular}

\section{Relevance ratio of multidrug-resistant organisms}

The proportion of colonization and infection with MDRO in critically ill patients at the time of ICU admission and ICU discharge every month is shown in Fig. 2. Among the total patients included in the study, the proportion of MDRO isolates decreased from 36 to $13 \%$ at the time of ICU admission and declined from 48 to $29 \%$ at the time of ICU discharge (both $p<0.001$ ). Proportion of MDRO isolates of patients who admitted into ICU directly from emergency department, which indicate that patients directly colonized from the community, decreased from 40 to $18 \%(p<0.001)$. Proportion of MDRO isolates of patients who transferred into ICU form other wards decrease from 34 to $11 \%(p<0.001)$. However, the proportion of MDRO isolates in patients from different departments decreased in varying degrees, even in some departments in which DDD was not decreased, such as in the thoracic surgery department (Table 3 ).

There were 802 and 992 samples for culture in phase 1 and phase 2 at ICU admission, respectively. Table 4 displays the microorganisms associated with colonization or infection at the time of ICU admission and ICU discharge. Among the $273 \mathrm{MDRO}$ present at the time of ICU admission, $75(27.4 \%)$ were MRSA and $83(30.4 \%)$ were $A$. baumannii, which is the highest rate among Grampositive and Gram-negative bacterium, respectively. When patients were discharged from the ICU, the rate of MRSA decreased from 27.4 to $20.4 \%$; however, A. baumannii infection increased from 30.4 to $40.7 \%$, whereas other bacterium did not change significantly (Table 4 ).

\section{Correlation between antimicrobial consumption and multidrug-resistant organisms}

As the antibiotic consumption decreased after antibacterial drug clinical application management, the proportion of colonization and infection with MDRO at the time of ICU admission correspondingly decreased. The 


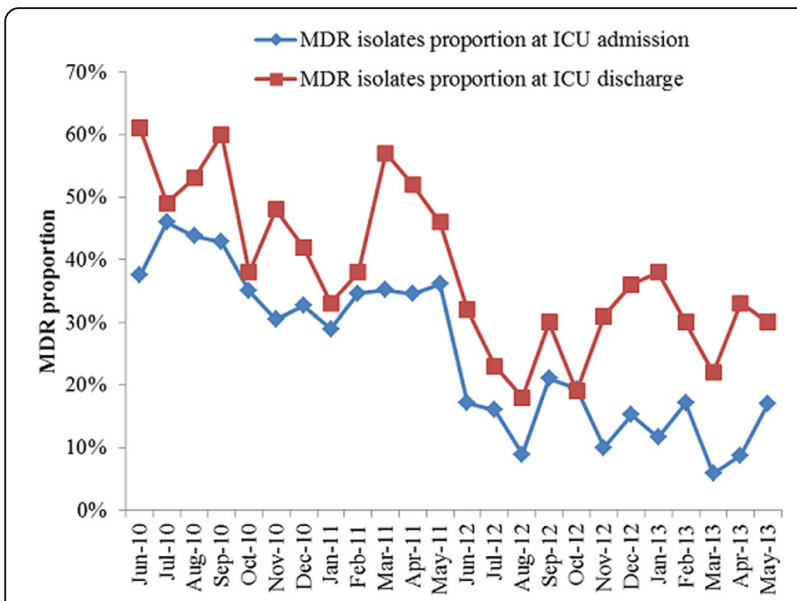

Fig. 2 Proportion of patient colonization of infection with MDRO at the time of ICU admission and discharge decreased after antimicrobial clinical application management

total consumption of antibacterial agents each month during the study period had a significant positive relationship with colonization and infection with MDRO in critically ill patients at the time of ICU admission each month $\left(\mathrm{R}^{2}=0.7858, p<0.001\right)$ (Fig. 3).

The DDD in the ICU may affect the MDRO proportion of critically ill patients at the time of ICU discharge. Therefore, we analyzed the relationship between these two factors. Although the MDRO rate decreased after the intervention, there was no significant relationship between the MDRO isolates and the DDD in the ICU $\left(\mathrm{R}^{2}=0.1085, p=0.116\right)$ (Fig. 4a). However, there was significant relationship between an increased MDRO rate from admission to discharge and the DDD in the ICU $\left(\mathrm{R}^{2}=0.1826, p=0.038\right)$ (Fig. $\left.4 \mathrm{~b}\right)$.

\section{Correlation between consumption of carbapenem and resistance of $\mathrm{A}$. baumannii to carbapenem}

A. baumannii was the most common MDRO in our study, and most $A$. baumannii are resistant to carbapenem. Therefore, we calculated the numbers of patients whose colonization or infection with $A$. baumannii were resistant to carbapenem at the time of ICU admission and discharge every month before and after the antimicrobial management. We also calculated the total carbapenem consumption in our hospital and in the ICU during the three-year study period. We analyzed the correlation between the consumption of carbapenem and the resistance of $A$. baumannii to carbapenem.

The proportion of patients colonized or infected with $A$. baumannii resistant to carbapenem at the time of ICU admission and discharge is presented in Fig. 5. The carbapenem consumption in our hospital and in the ICU during the three-year period decreased from 10.1 to $5.7 \%$ at the time of ICU admission and from 20.5 to $15.4 \%$ at the time of ICU discharge after the intervention. However, the DDD of carbapenem in the hospital and in the ICU did not significantly change after the intervention $(1.33 \pm$ 0.33 vs. $1.26 \pm 0.28, p=0.446$ and $18.2 \pm 5.4$ vs. $23.2 \pm 7.7$, $p=0.573$, respectively). There was no significant relationship between the consumption of carbapenem in the hospital and resistance of $A$. baumannii to carbapenem at the time of ICU admission $\left(\mathrm{R}^{2}=0.0436, p=0.393\right)$ (Fig. 5a). Similar results were found between the consumption of carbapenem in the ICU and the resistance of

Table 3 Proportion of patients colonized or infected with MDRO before and after antimicrobial stewardship

\begin{tabular}{|c|c|c|c|c|}
\hline Department & All patients (978) & Before management (433) & After management (545) & $p$ value \\
\hline All, n (\%) & $225(23.01)$ & $154(35.57)$ & $71(13.03)$ & $<0.001$ \\
\hline Emergency, n (\%) & $86(23.76)$ & $54(40.91)$ & $32(17.68)$ & $<0.001$ \\
\hline Surgery, n (\%) & $91(19.24)$ & $68(33.17)$ & $23(8.58)$ & $<0.001$ \\
\hline Neurosurgery, n (\%) & $11(13.75)$ & $5(29.41)$ & $6(9.52)$ & 0.035 \\
\hline Thoracic surgery, n (\%) & $12(10.52)$ & $11(20.37)$ & $1(1.67)$ & 0.001 \\
\hline General surgery, n (\%) & $35(26.92)$ & $28(40.00)$ & $7(11.67)$ & $<0.001$ \\
\hline Urology surgery, n (\%) & $10(28.57)$ & $8(38.10)$ & $2(14.29)$ & 0.252 \\
\hline Orthopedics, n (\%) & $8(18.18)$ & $8(40.00)$ & $0(0)$ & 0.001 \\
\hline Other, n (\%) & $13(18.57)$ & $6(26.09)$ & $7(14.89)$ & 0.258 \\
\hline Medicine, n (\%) & $50(27.47)$ & $34(30.52)$ & $16(16.84)$ & 0.033 \\
\hline Respiratory medicine, n (\%) & $10(32.26)$ & $8(50.00)$ & $2(13.33)$ & 0.054 \\
\hline Neurology, n (\%) & $13(38.23)$ & $8(42.11)$ & $5(33.33)$ & 0.728 \\
\hline Cardiology, n (\%) & $7(15.22)$ & $5(21.73)$ & $2(8.70)$ & 0.218 \\
\hline Other, n (\%) & $20(25.00)$ & $13(35.14)$ & $7(16.28)$ & 0.062 \\
\hline
\end{tabular}


Table 4 Microorganisms isolation at the time of ICU admission and discharge

\begin{tabular}{|c|c|c|}
\hline & ICU admission & ICU discharge \\
\hline All microorganisms & $1054(100)$ & $1040(100)$ \\
\hline Multidrug-resistant bacteria ${ }^{a}$ & $273(25.9)$ & $369(35.5)$ \\
\hline $\begin{array}{l}\text { Methicillin-resistant } \\
\text { Staphylococcus aureus }\end{array}$ & $75(27.4)$ & $74(20.1)$ \\
\hline $\begin{array}{l}\text { Vancomycin-resistant } \\
\text { enterococci }{ }^{\mathrm{b}}\end{array}$ & $10(3.6)$ & $8(2.2)$ \\
\hline Pseudomonas aeruginosa ${ }^{b}$ & $12(4.4)$ & $18(4.9)$ \\
\hline Acinetobacter baumannii ${ }^{b}$ & $83(30.4)$ & $150(40.7)$ \\
\hline Escherichia colib & $24(8.8)$ & $27(7.3)$ \\
\hline Klebsiella oxytocab & $15(5.5)$ & $24(6.5)$ \\
\hline Enterobacter cloacae $^{b}$ & $5(1.8)$ & $3(0.5)$ \\
\hline Proteus species ${ }^{b}$ & $1(0.4)$ & $4(1.1)$ \\
\hline Other $^{\mathrm{b}}$ & $46(16.8)$ & $59(16.0)$ \\
\hline $\begin{array}{l}\text { Non-multidrug-resistant } \\
\text { bacteria }^{\mathrm{a}}\end{array}$ & $781(74.1)$ & $671(64.5)$ \\
\hline Staphylococcus epidermidis ${ }^{c}$ & $375(45.7)$ & $307(45.8)$ \\
\hline Staphylococcus haemolyticus ${ }^{c}$ & $33(4.2)$ & $32(4.8)$ \\
\hline $\begin{array}{l}\text { Methicillin-sensitive } \\
\text { Staphylococcus aureus }\end{array}$ & $49(6.3)$ & $51(7.6)$ \\
\hline Enterococcus species ${ }^{c}$ & $18(2.6)$ & $21(3.1)$ \\
\hline Pseudomonas aeruginosa ${ }^{c}$ & $15(1.9)$ & $13(1.9)$ \\
\hline Acinetobacter baumanniic & $10(1.3)$ & $11(1.6)$ \\
\hline Escherichia colic & $26(3.3)$ & $20(3.0)$ \\
\hline Klebsiella oxytoca ${ }^{c}$ & $100(12.8)$ & $84(12.5)$ \\
\hline Enterobacter cloacae ${ }^{c}$ & $13(1.7)$ & $12(1.8)$ \\
\hline Proteus species $^{c}$ & $7(0.9)$ & $6(0.9)$ \\
\hline Others $^{c}$ & $140(17.9)$ & $111(16.5)$ \\
\hline
\end{tabular}

Data were presented by counts and percentage

a Percentage equals the counts of the item divided by all microorganisms

${ }^{\text {b} P e r c e n t a g e ~ e q u a l s ~ t h e ~ c o u n t s ~ o f ~ t h e ~ i t e m ~ d i v i d e d ~ b y ~ m u l t i d r u g-r e s i s t a n t ~ b a c t e r i a ~}$

${ }^{c}$ Percentage equals the counts of the item divided by non-multidrug-resistant bacteria ICU intensive care unit

A. baumannii to carbapenem at the time of ICU discharge $\left(\mathrm{R}^{2}=0.0798, p=0.181\right)$ (Fig. 5b).

\section{The incidence of HCAls before and after antimicrobial stewardship}

During the intervention, we also monitored the incidence of ventilator associated pneumonia (VAP), catheter related blood stream infection (CRBSI) and catheter associated urinary tract infection (CAUTI), which were the most common of health-care-associated infections (HCAIs) in our ICU. We found that the incidence of VAP was significantly decreased after the intervention. However, the incidence of CRBSI and CAUTI did not change after the antimicrobial stewardship (Additional file 2: Table S2).

\section{Discussion}

Infection with MDRO is frequently observed during clinical treatment [15-17], especially in critically ill patients [18]. Preventing infection with MDRO is very important to our clinic workers. To the best of our knowledge, this is the first study concerning the effect of the antimicrobial stewardship program of Chinese $\mathrm{MOH}$ on colonized or infected with MDRO in critically ill patients. In the present study, we found that DDD in the hospital and in most departments decreased significantly after the implementation of the antimicrobial stewardship.

Consequently, the MDRO isolation in critically ill patients correspondingly decreased when they admitted into ICU. However, we found a low relationship between DDD in the ICU and increased MDRO rate from ICU admission to discharge. Moreover, we did not find a positive relationship between DDD in the ICU and MRDO at ICU or between the DDD of carbapenem and carbapenem-resistant $A$. baumannii.

Antimicrobial stewardship can reduce the DDD in the entire hospital and in the majority of the departments. After the intervention, we found that the rational use of antibiotics had improved. In the preventative use of antibiotics in clean operation, for example, the percentage decreased from nearly 100 to 35\%, which was in line with previous data [19]. Consequently, the DDD was decreased significantly from 96 to 65 in our hospital and was decreased in most departments (Table 2). Our results confirmed the previous results in China [20]. However, our DDD is still higher than the standard claimed by the policy. Therefore, we should conscientiously execute this policy to improve antibiotic use.

Antimicrobial stewardship can help to decrease the MDRO isolation in critically ill patients. Along with the decrease in antimicrobial consumption in our study, the proportion of colonization or infection with MDRO in critically ill patients decreased significantly to $13 \%$. Furthermore, we found a good positive correlation between antibiotic consumption and MDRO in our hospital (Fig. 3). Previous studies demonstrated the association between antimicrobial use and MDRO detection [2-4, 21]. Bassetti et al. found a significant correlation between antibiotic consumption and increased resistance for K. pneumonia [21]. A recent study in China demonstrated that increased consumption of carbapenem may contribute to the development of resistance in A. baumannii to imipenem, meropenem, and other antimicrobials [4]. However, Most previous studies were demonstrated that with the increased consumption of antimicrobials, the MDRO increased correspondingly [2-4, 21-23]. There was only the some correlation between MDRO and antimicrobial consumption. Whether the decrease antimicrobial consumption could reduce the MDRO was not very clear. 


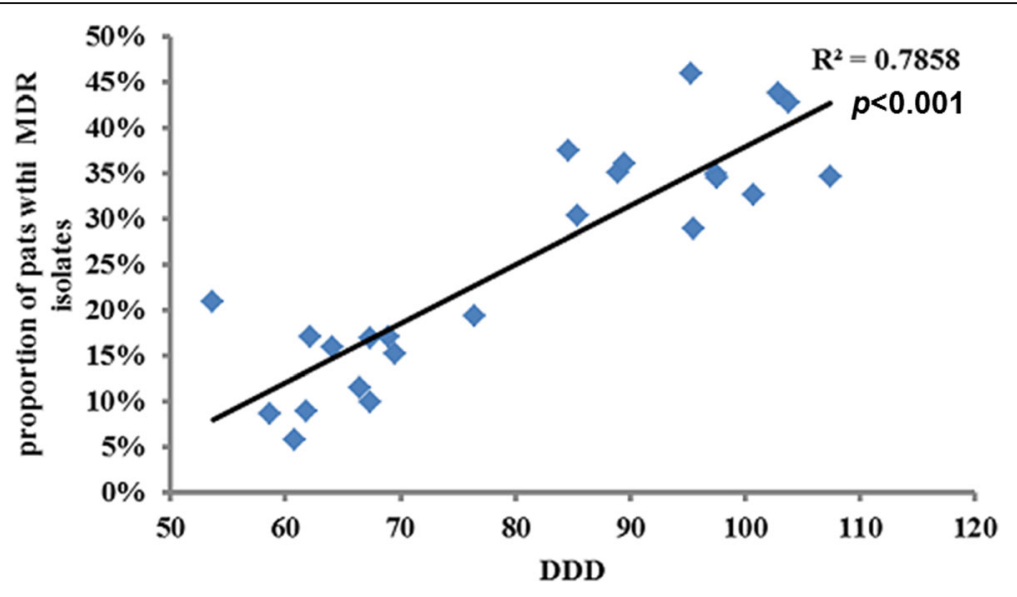

Fig. 3 A significant positive relationship between the proportion of critically ill patient colonization or infection with MDRO at the time of ICU admission monthly and total antimicrobial consumption in our hospital monthly $\left(R^{2}=0.7858, p<0.001\right)$

Interestingly, we found that the proportion of patients colonized or infected with MDRO decreased along with reduced consumption of antibiotics.

In some departments, such as the thoracic surgery department, the DDD did not decrease; however, the MDRO isolation rates in this department decreased significantly in critically ill patients. Several reasons may explain this decrease. First, DDD did not equal to rational application of antimicrobials. Moreover, the

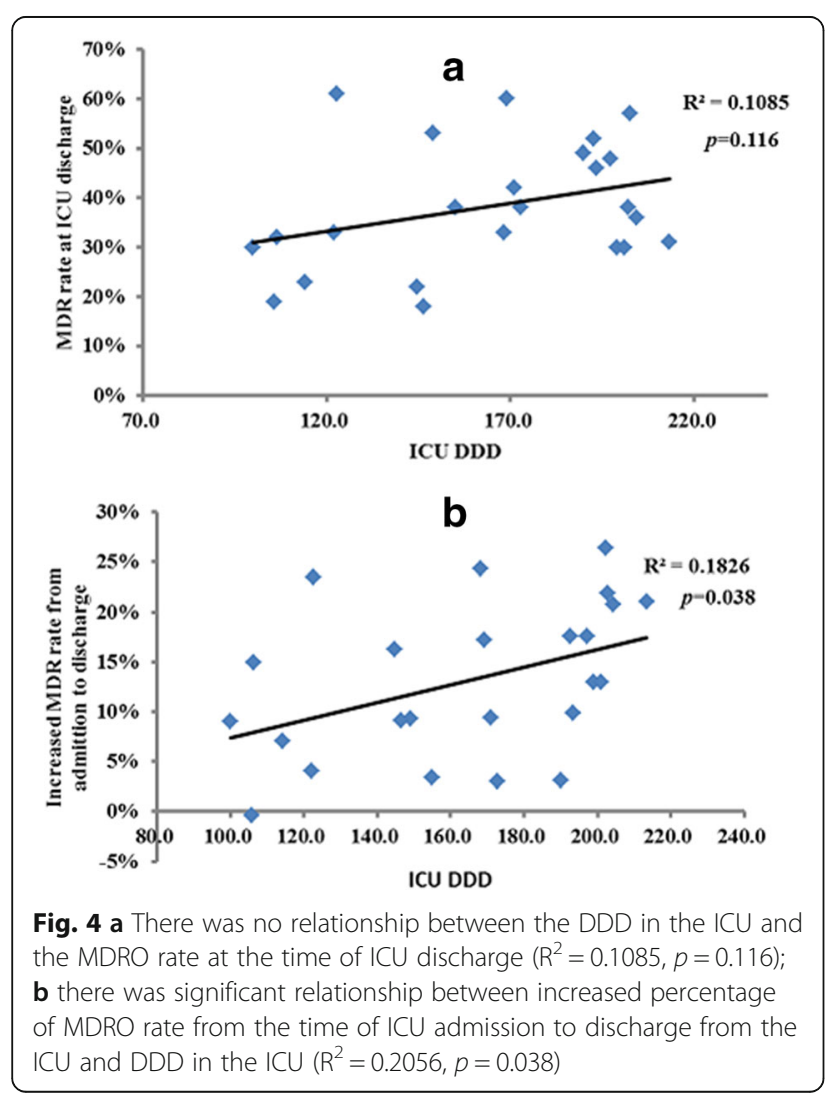

sample from such departments was small and could not achieve significance. Finally, MDRO are easily transmitted from patient to patient through hand contact with doctors, nurses and hospital workers [22, 23]. Additionally, critically ill patients were transferred several times to different departments before being initially admitted into the ICU. Therefore, the incidence of MDRO isolates

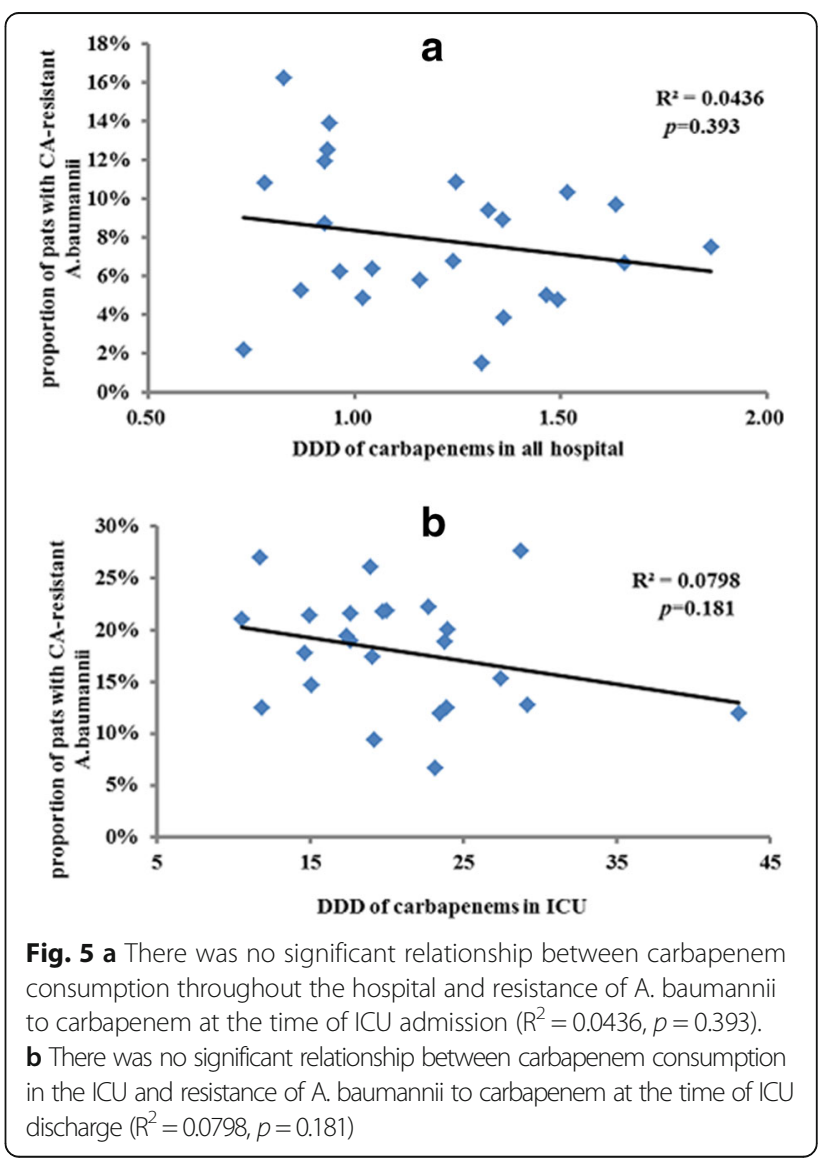


in patients from different departments and throughout the hospital decreased, although DDD did not decrease.

Carbapenem-resistant $A$. baumannii is a serious problem in the clinic, especially in the ICU $[24,25]$. Although the DDD of carbapenem did not decrease in the entire hospital and in the ICU, the carbapenem-resistant $A$. baumannii isolates decreased both at the time of ICU admission and at ICU discharge. Moreover, we did not find a good relationship between carbapenem consumption and carbapenem-resistant A. baumannii. Because previous studies have examined whether carbapenem restriction can reduce the rates of carbapenem-resistant $A$. baumannii and have had inconsistent findings [4, 26], further explorations are warranted to confirm these results.

Our study has several limitations. First, this study was performed in a single center. Because drug resistance rates vary among hospitals and units, the results may not be representative and reproducible in other institutions. However, in one hand, antibiotic overuse is common in China [10], and antimicrobial stewardship can improve the use of antibiotics. In another hand, the results of antimicrobial consumption in our study was in line with previous report [20]. Additionally, studies have demonstrated that the rational use of antibiotics could prevent antimicrobial resistance [27]. Therefore, we believe that our study can benefit other hospitals.

Second, we did not analyze the individual risk factors for colonization or infection with resistant microorganisms. Factors that would affect the MDRO isolation, such as hand hygiene, isolating the high-risk patients, and taking precautions, were not analyzed. However, during this pre-post study period in our hospital, we had taken executive infection control measures. We found that the compliance of hand hygiene did not significantly change in our department. Therefore, we believe that the decrease of DDD is an important factor for reducing the rate of MDRO. We also plan to investigate and analyze the risk factors concerning infection and MDRO.

Third, we did not screen the MDRO since rectal swabs, and the proportion of undetected MDRO colonizations could consequently be high. However, the policy of screen was the same during the two periods, therefore, it will not be influence our results hugely.

Finally, our study did not address the issue of antibiotic use and resistance in the community. The consumption of antimicrobials in agricultural industry may also promote antibiotic resistance $[28,29]$. However, the MDRO isolation of patients who arrived from emergency department also decreased from 40 to $18 \%$ and may not affect our results.

\section{Conclusion}

The present study has demonstrated that the antibacterial drug clinical application management established by
China's Ministry of Public Health can improve the rational use of antibiotics and reduce the antibiotic consumption. Consequently, with the decrease of DDD in the hospital, the proportion of colonization or infection with MDRO correspondingly decreased. There was a significant positive relationship between the proportion of all critically ill patients colonized or infected with MDRO at the time of ICU admission and the DDD of the all hospital. However, this relationship did not exist in the local department or DDD of the individual medication.

\section{Additional files}

Additional file 1: Table S1. The category of antibiotics before and after antimicrobial stewardship. (DOCX $16 \mathrm{~kb}$ )

Additional file 2: Table S2. The incidence of HCAls before and after antimicrobial stewardship. Incidence of VAP, CRBSI and CAUTI were defined as the number of VAP, CRBSI and CAUTI patients per 1000 ventilation days, per 1000 central venous catheter days and per 1000 urine-catheter days, respectively. (DOCX $15 \mathrm{~kb}$ )

\section{Abbreviations}

CAUTI: Catheter associated urinary tract infection; CRBSI: Catheter related blood stream infection; DDD: Defined daily dose; ESBL: Extended-spectrum $\beta$-lactamase; HCAls: Health-care-associated infections; ICU: Intensive care unit; MDRO: Multidrug-resistant organism; MIC: Minimal inhibitory concentration; $\mathrm{MOH}$ : Ministry of health; MRSA: Methicillin-resistant Staphylococcus aureus; VAP: Ventilator associated pneumonia; VRE: Vancomycin-resistant enterococci; WHO: World Health Organization

\section{Acknowledgements}

We thank all patients and clinicians from the Zhongda Hospital in Nanjing, China, for their cooperation and participation in the study; Yaxiang Shi (Computer Information Center, Zhongda hospital, School of Medicine, Southeast University) for getting the consumption of antibiotics of our hospital. This work was supported by major special projects of Ministry of Health of China (grant number: 201202011), and fund of the key discipline of Jiangsu province (grant number KJXW11.3.).

\section{Funding}

This work was supported by major special projects of Ministry of Health of China (grant number: 201202011), and fund of the key discipline of Jiangsu province (grant number KJXW11.3.).

\section{Availability of data and materials}

The datasets used and/or analysed during the current study available from the corresponding author on reasonable request.

\section{Authors' contributions}

XDM, JFX, YY and HBQ designed the study. XDM, JFX, ZWG, and CSY collected most of the data. XDM, JFX, FMG, YZH and HBQ interpreted and analyzed the data. All members participated in the drafting and revision of the manuscript and added substantive intellectual contributions. All authors read and approved the final manuscript.

\section{Competing interests}

All contributing authors declare that there is no conflict of interest. No competing interests exist in this study due to commercial or other associations (e.g.pharmaceutical stock ownership, consultancy, advisory board membership, relevant patents, or research funding). No competing interests exist in the submission of this manuscript, and the manuscript is approved by all of the authors for publication.

Consent for publication

Not applicable. 


\section{Ethics approval and consent to participate}

The protocol of our study was approved by Nanjing Zhongda Hospital Institutional Ethics Committee (Approval Number: 2011ZDLL012.0). Written informed consent was obtained from the patients or next of kin.

\section{Author details}

'School of Medicine and Health Management, Tongji Medical College of Huazhong University of Science and Technology, Wuhan 430074, China. ${ }^{2}$ Department of Critical Care Medicine, Nanjing Zhongda Hospital, School of Medicine, Southeast University, Nanjing 210009, China. ${ }^{3}$ Department of Pharmacy, Nanjing Zhongda Hospital, School of Medicine, Southeast University, Nanjing 210009, China.

\section{Received: 30 March 2016 Accepted: 21 November 2016}

Published online: 25 November 2016

\section{References}

1. Williams RJ, Heymann DL. Containment of antibiotic resistance. Science. 1998;279(5354):1153-4

2. Lai CC, Wang CY, Chu CC, Tan CK, Lu CL, Lee YC, Huang YT, Lee PI, Hsueh PR. Correlation between antibiotic consumption and resistance of gramnegative bacteria causing healthcare-associated infections at a University Hospital in Taiwan from 2000 to 2009. J Antimicrob Chemother. 2011;66(6): 1374-82.

3. Muraki Y, Kitamura M, Maeda Y, Kitahara T, Mori T, Ikeue H, Tsugita M, Tadano K, Takada K, Akamatsu T, et al. Nationwide surveillance of antimicrobial consumption and resistance to Pseudomonas aeruginosa isolates at 203 Japanese hospitals in 2010. Infection. 2013:41(2):415-23.

4. Cao J, Song W, Gu B, Mei YN, Tang JP, Meng L, Yang CQ, Wang H, Zhou H. Correlation between carbapenem consumption and antimicrobial resistance rates of Acinetobacter baumannii in a university-affiliated hospital in China. J Clin Pharmacol. 2013;53(1):96-102.

5. Dellit TH, Owens RC, McGowan Jr JE, Gerding DN, Weinstein RA, Burke JP, Huskins WC, Paterson DL, Fishman NO, Carpenter CF, et al. Infectious diseases society of America and the society for healthcare epidemiology of America guidelines for developing an institutional program to enhance antimicrobial stewardship. Clin Infect Dis. 2007:44(2):159-77.

6. World Health Organization (WHO). WHO global strategy for containment of antimicrobial resistance. 2010. http://www.who.int/csr/resources/ publications/drugresist/en/EGlobal_Strat.pdf. Accessed Apr 2014.

7. Maechler F, Schwab F, Geffers C, Meyer E, Leistner R, Gastmeier P. Antibiotic stewardship in Germany: a cross-sectional questionnaire survey of 355 intensive care units. Infection. 2014;42(1):119-25.

8. Jensen US, Skjot-Rasmussen L, Olsen SS, Frimodt-Moller N, Hammerum AM, Group DS. Consequences of increased antibacterial consumption and change in pattern of antibacterial use in Danish hospitals. J Antimicrob Chemother. 2009;63(4):812-5.

9. Haug JB, Berild D, Walberg M, Reikvam A. Increased antibiotic use in Norwegian hospitals despite a low antibiotic resistance rate. J Antimicrob Chemother. 2011;66(11):2643-6.

10. Liang X, Jin C, Wang L, Wei L, Tomson G, Rehnberg C, Wahlstrom R, Petzold M. Unnecessary use of antibiotics for inpatient children with pneumonia in two counties of rural China. Int J Clin Pharm. 2011;33(5):750-4.

11. Zhang R, Eggleston K, Rotimi V, Zeckhauser RJ. Antibiotic resistance as a global threat: evidence from China, Kuwait and the United States. Globalization Health. 2006;2:6.

12. Xiao YH, Giske CG, Wei ZQ, Shen P, Heddini A, Li L. Epidemiology and characteristics of antimicrobial resistance in China. Drug Resist Updat. 2011:14(4-5):236-50.

13. Preventing Healthcare-associated Infections. http://www.cdc.gov/hai/ prevent/prevention.html. Accessed Apr 2014

14. Magiorakos AP, Srinivasan A, Carey RB, Carmeli Y, Falagas ME, Giske CG, Harbarth S, Hindler JF, Kahlmeter G, Olsson-Liljequist B, et al. Multidrugresistant, extensively drug-resistant and pandrug-resistant bacteria: an international expert proposal for interim standard definitions for acquired resistance. Clin Microb Infect. 2012;18(3):268-81.

15. Boucher HW, Talbot GH, Bradley JS, Edwards JE, Gilbert D, Rice LB, Scheld M, Spellberg B, Bartlett J. Bad bugs, no drugs: no ESKAPE! an update from the infectious diseases society of America. Clin Infect Dis. 2009;48(1):1-12.

16. Heddini A Cars O, Qiang S, Tomson G. Antibiotic resistance in China-a major future challenge. Lancet. 2009;373(9657):30.
17. Cao B, Zhao CJ, Yin YD, Zhao F, Song SF, Bai L, Zhang JZ, Liu YM, Zhang YY, Wang $\mathrm{H}$, et al. High prevalence of macrolide resistance in Mycoplasma pneumoniae isolates from adult and adolescent patients with respiratory tract infection in China. Clin Infect Dis. 2010;51(2):189-94.

18. Wroblewska MM, Rudnicka J, Marchel H, Luczak M. Multidrug-resistant bacteria isolated from patients hospitalised in intensive care units. Int J Antimicrob Agents. 2006;27(4):285-9.

19. Xiao Y, Zhang J, Zheng B, Zhao L, Li S, Li L. Changes in Chinese policies to promote the rational use of antibiotics. PLoS Med. 2013:10(11):e1001556.

20. China MoH (10 March 2013) Available: http://www.moh.gov.cn/mohbgt/ s3582/201205/54651.shtml. News Conference on May 8, 2012. Accessed Apr 2014

21. Bassetti M, Cruciani M, Righi E, Rebesco B, Fasce R, Costa A, Molinari MP Mengoli C, Bobbio Pallavicini F, Viscoli C. Antimicrobial use and resistance among gram-negative bacilli in an Italian intensive care unit (ICU). J Chemother. 2006;18(3):261-7.

22. Scott P, Deye G, Srinivasan A, Murray C, Moran K, Hulten E, Fishbain J, Craft $D$, Riddell $S$, Lindler $L$, et al. An outbreak of multidrug-resistant Acinetobacter baumannii-calcoaceticus complex infection in the US military health care system associated with military operations in Iraq. Clin Infect Dis. 2007:44(12):1577-84

23. Sanchez Garcia M, De la Torre MA, Morales G, Pelaez B, Tolon MJ, Domingo S, Candel FJ, Andrade R, Arribi A, Garcia N, et al. Clinical outbreak of linezolid-resistant Staphylococcus aureus in an intensive care unit. JAMA. 2010;303(22):2260-4.

24. Munoz-Price LS, Weinstein RA. Acinetobacter infection. N Engl J Med. 2008;358(12):1271-81.

25. Peleg AY, Hooper DC. Hospital-acquired infections due to gram-negative bacteria. N Engl J Med. 2010;362(19):1804-13.

26. Sistanizad M, Kouchek M, Miri M, Goharani R, Solouki M, Ayazkhoo L, Foroumand M, Mokhtari M. Carbapenem restriction and its effect on bacterial resistance in an intensive care unit of a teaching hospital. Iran J Pharm Res. 2013;12(3):503-9.

27. Chen IL, Lee CH, Su LH, Tang YF, Chang SJ, Liu JW. Antibiotic consumption and healthcare-associated infections caused by multidrugresistant gram-negative bacilli at a large medical center in Taiwan from 2002 to 2009: implicating the importance of antibiotic stewardship. PLoS One. 2013;8(5):e65621.

28. Levy SB. The 2000 garrod lecture. Factors impacting on the problem of antibiotic resistance. J Antimicrob Chemother. 2002;49(1):25-30.

29. Fey PD, Safranek TJ, Rupp ME, Dunne EF, Ribot E, Iwen PC, Bradford PA, Angulo FJ, Hinrichs SH. Ceftriaxone-resistant salmonella infection acquired by a child from cattle. N Engl J Med. 2000;342(17):1242-9.

\section{Submit your next manuscript to BioMed Central and we will help you at every step:}

- We accept pre-submission inquiries

- Our selector tool helps you to find the most relevant journal

- We provide round the clock customer support

- Convenient online submission

- Thorough peer review

- Inclusion in PubMed and all major indexing services

- Maximum visibility for your research

Submit your manuscript at www.biomedcentral.com/submit
Biomed Central 